\title{
An Advanced Preamplifier for Highly Segmented Germanium Detectors
}

\author{
A. Pullia, F. Zocca, and G. Pascovici
}

\begin{abstract}
We present a fast low-noise hybrid charge-sensitive preamplifier for germanium position-sensitive $\gamma$-ray detectors. In conjunction with a bulky 36-fold segmented detector it provided an excellent resolution of $1.71 / 1.77 \mathrm{keV}$ fwhm on the 1.17/1.33 $\mathrm{MeV}^{60}$ Co lines. The preamplifier rise time, as measured at the test bench, is as fast as $\mathbf{7 . 5} \mathbf{~ n s , ~ w i t h ~ a ~ d e t e c t o r ~ c a p a c i t a n c e ~}$ of $21 \mathrm{pF}$ and with a $5 \mathrm{~m} 50 \Omega$ twisted-pair cable connected at its output. The dynamic range of the preamplifier input stage is as large as $92 \mathrm{~dB}$, ranging from $0.275 \mathrm{fC}$ to $9.9 \mathrm{pC}$, i.e., from $5 \mathrm{keV}$ to $180 \mathrm{MeV}$ in terms of photon energy. On signals larger than $2 \mathrm{MeV}$ a fast reset $(\sim 10 \mathrm{MeV} / \mu \mathrm{s})$ is enforced to reduce the system dead time. An estimate of the amplitude of such large signals is derived from the reset time, still obtaining a high resolution. Using this technique we achieved an energy resolution of $0.3 \%$ at $16.7 \mathrm{MeV}$.
\end{abstract}

Index Terms-Charge-sensitive preamplifier, gamma ray spectroscopy, germanium detector.

\section{INTRODUCTION}

A NEW generation of nuclear-physics experiments with radioactive ion beams is foreseen in the near future. In these experiments $4 \pi$ arrays of monolithic multi-electrode positionsensitive germanium detectors will be used for spectroscopy and tracking of $\gamma$ rays [1], [2]. In these experiments besides having a low noise, a fast rise time is required because the shape of the leading edge of the preamplifier signal is a key signature of the physical positions of the $\gamma$-photon interaction points inside the detector [3]. A large dynamic range is also required for a class of experiments where giant-dipole resonances can produce $\gamma$ photons in the $20 \mathrm{MeV}$ range [4]. Another key requirement is a reduced dead time in the presence of a background of charged particles (pions, kaons), in the 10 to $100 \mathrm{MeV}$ energy range.

We have developed a dedicated preamplifier, optimized in such a way to feature low noise, a fast rise time, and a large dynamic range. In addition the preamplifier is equipped with a time-variant device which quickly resets the circuitry on large signals in excess of a few $\mathrm{MeV}$. This device substantially reduces the dead time caused by large signals or random walks that could saturate the output buffer or the ADC voltage range.

Two versions have been realized. One is optimized for cathode detector electrodes. One other for anode detector electrodes. Both include a fast differential buffer for low-noise signal transmission to a remote ADC.

Manuscript received December 4, 2005; revised June 23, 2006.

A. Pullia and F. Zocca are with the University of Milan, Department of Physics and Istituto Nazionale di Fisica Nucleare, I-20133 Milano, Italy.

G. Pascovici is with the University of Koeln, Institüt für Kernphysik, D-50937 Koeln, Germany.

Digital Object Identifier 10.1109/TNS.2006.880402

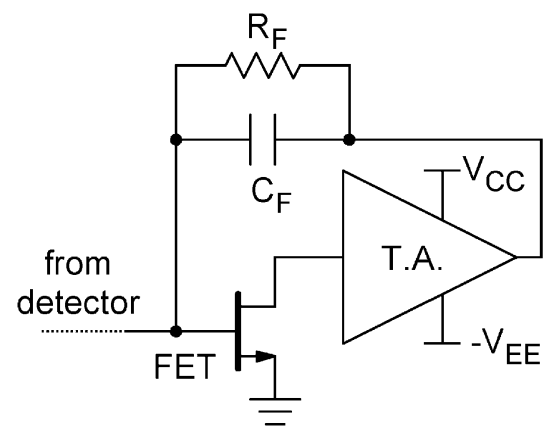

Fig. 1. Structure of the charge-sensing stage. The chosen values for the feedback devices are $\mathrm{C}_{\mathrm{F}}=1 \mathrm{pF}$ and $\mathrm{R}_{\mathrm{F}}=1 \mathrm{G} \Omega$.

\section{PREAmPLifiER STRUCTURe}

The charge-sensing stage of the preamplifier comprises $(a)$ an input Field-Effect Transistor and a feedback passive network mounted in the cryostat as close as possible to the detector electrode, and (b) a Transimpedance Amplifier (TA) operated at room temperature outside the cryostat, as shown in Fig. 1. In Fig. 2 the simplified schematic diagrams of the realized transimpedance amplifiers are shown, for the cases of cathode and anode detector signals. In both cases a 'folded cascode' structure is adopted for the gain stage, with the unusual solution of using two cascaded bipolar transistors in the common-base path (T1/T2). This solution has these advantages: (i) it yields a substantial increase of the loop gain and (ii) it permits to maximize the negative/positive output-voltage range for either cathode [Fig. 2(a)] or anode [Fig. 2(b)] detector signals. The drawback of the double-transistor cascode solution is an increase of the electronic noise, which, however, may be made negligible by properly biasing the circuit structure. The output voltage range of the TA is about $10 \mathrm{~V}$ for either cathode or anode signal versions. As the closed loop gain is $\sim 55 \mathrm{mV} / \mathrm{MeV}$ $\left(\mathrm{C}_{\mathrm{F}}=1 \mathrm{pF}\right)$ we obtain an energy range of $\sim 180 \mathrm{MeV}$. From experimental tests we found that the low-level discrimination threshold can be set down to $\sim 5 \mathrm{keV}$. So we get a dynamic range of as much as $92 \mathrm{~dB}$ for the integration stage.

To get a fast rise time we have used a fast input FET, mod. BF862, and broadband bipolar transistors, mod. BFT92 and BFR92, with a cut-off frequency in excess of $1 \mathrm{GHz}$ at the used collector bias currents. We have carefully chosen the collector current $\mathrm{I}_{\mathrm{C}}$ of the cascode transistors in order to guarantee both a low noise and a wide bandwidth, finding an optimum value for $\mathrm{I}_{\mathrm{C}}$ of $380 \mu \mathrm{A}$. A further increase of $\mathrm{I}_{\mathrm{C}}$ yields a higher noise and no significant improvement of the preamplifier bandwidth.

In Fig. 3 the simplified schematic diagram of the entire preamplifier (version for anode signals) is shown. The 

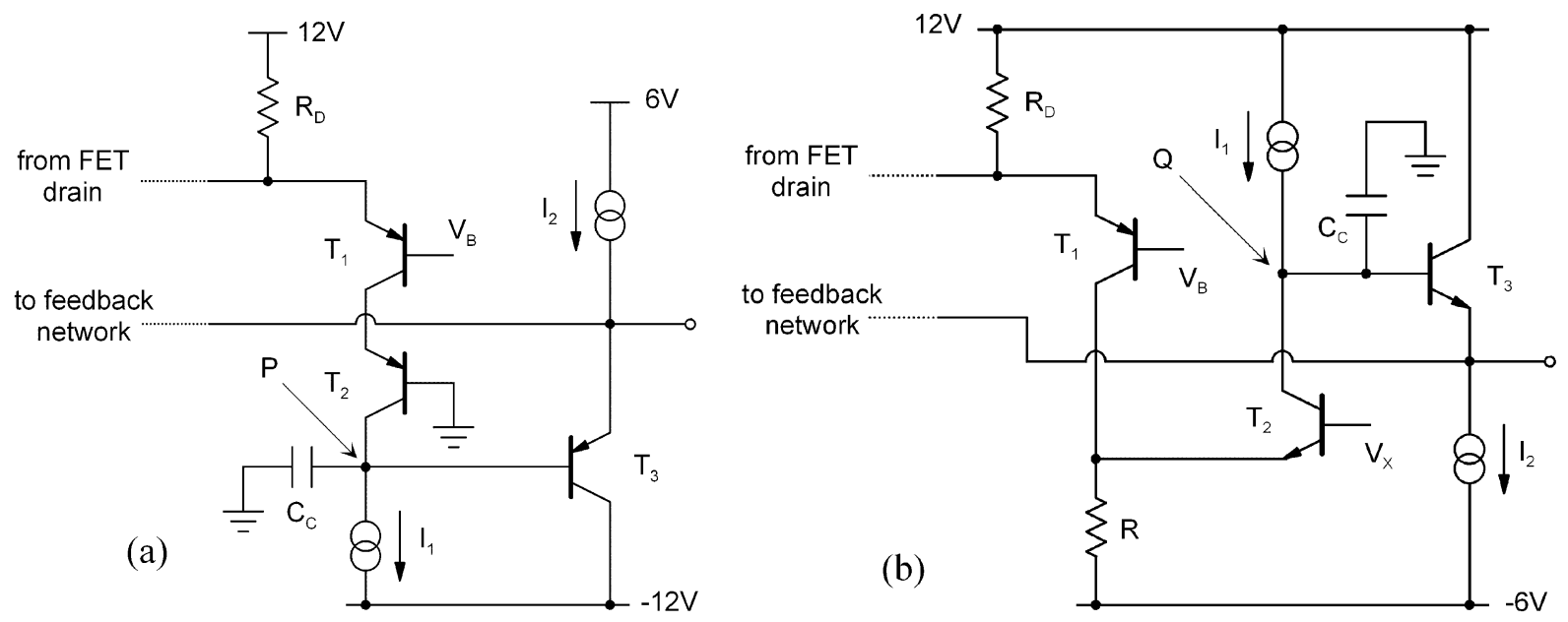

Fig. 2. Simplified schematic diagram of the realized transimpedance amplifier. (a) Case for cathode detector signals, or negative output voltage swing. (b) Case for anode detector signals, or positive output voltage swing. Capacitance $\mathrm{C}_{\mathrm{C}}$ is used to stabilize the feedback loop.

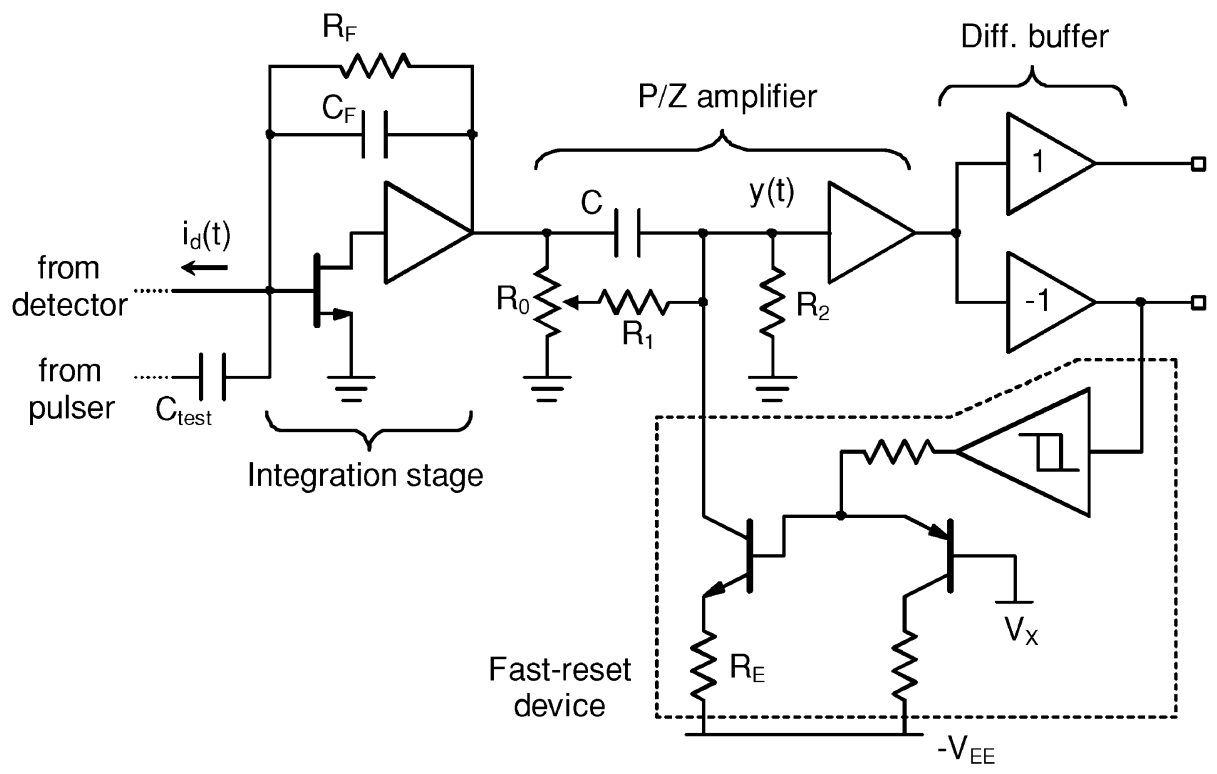

Fig. 3. Simplified schematic diagram of the preamplifier. It consists of an integration stage, a Pole/Zero compensation network, and a fast-reset circuitry which automatically switches on when the output voltage overcomes a preset threshold.

charge-sensitive stage of the preamplifier is followed by a Pole/Zero stage and a differential line driver. The differential signal transmission mode is useful to enhance the rejection to common-mode noise and disturbances picked up along the output cable. The signal can so be transmitted to a remote ADC module through a cable of several meters preserving its original quality, which makes the preamplifier suitable for noisy experimental environments. The fast-reset circuitry is put in evidence by a dashed line in the lower part of the schematic diagram. It consists of a Schmitt trigger comparator which switches on/off a temperature-compensated current sink. When a large signal arrives the current sink is switched on, which discharges capacitance $\mathrm{C}$ and quickly restores voltage $\mathrm{y}(t)$ to 0 . As soon as $y(t)$ (as well as the output voltage) reaches $0 \mathrm{~V}$, the current sink is switched off, and normal operation is restored [5].

\section{FeEdBACK LoOp ANALYSIS}

Maximization of the preamplifier loop gain is of primary importance in gamma-ray spectroscopy with germanium detectors. In fact the energy resolution obtainable with these detectors is as high as $0.2 \%$ at $1 \mathrm{MeV}$. In order to achieve and maintain such a good resolution an outstanding long-term gain stability of less than $0.2 \%$ is required. Provided that a high-quality feedback capacitance is used, the larger the loop gain and the better the preamplifier long-term gain stability.

The double-transistor cascode structure yields $(i)$ a larger overall load resistance at the cascode output, and (ii) a reduced Miller effect on the gate-to-drain capacitance of the input FET. Both of these features contribute to maximize the open-loop gain as well as the loop gain of the circuit, as is described next. 


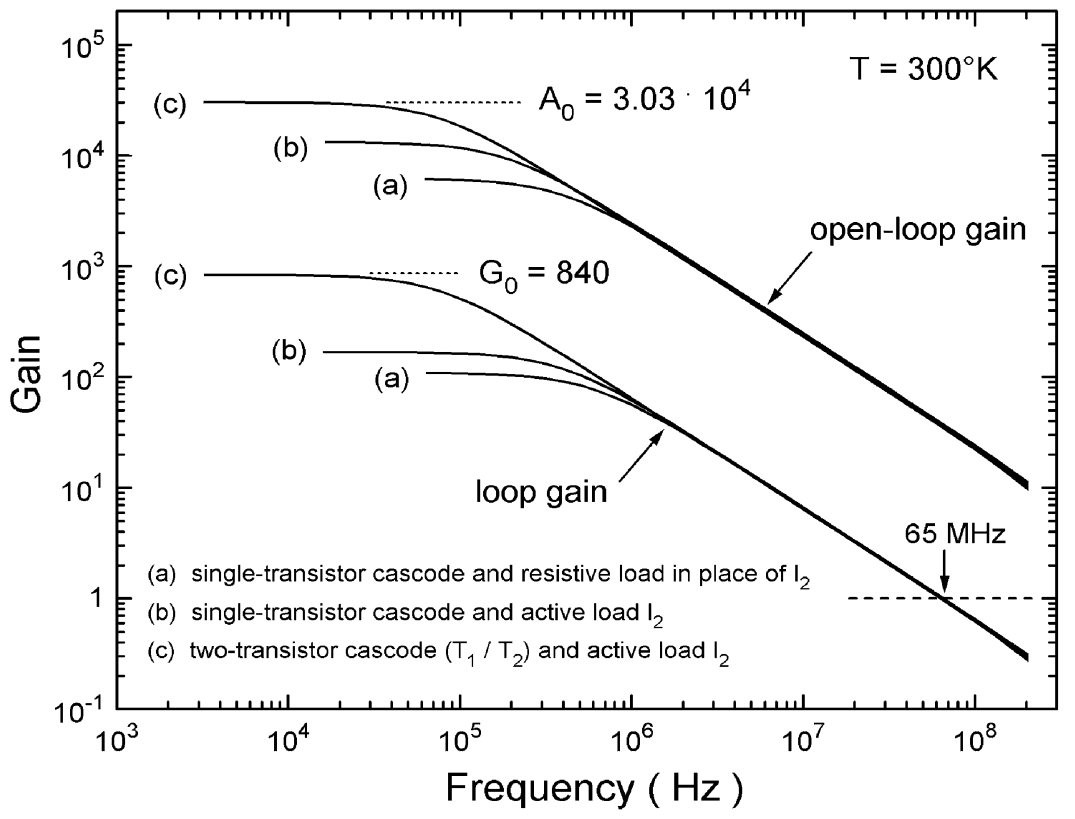

Fig. 4. Open loop gain and loop gain of the integration stage versus frequency. Three different cases are shown, which subsequently yield an improvement of the gain value at low frequency.

\section{A. Open-Loop Gain}

Let us consider the "gain node" of the circuit, i.e., respectively point $\mathrm{P}$ or $\mathrm{Q}$ in Figs. 2(a) and (b). At low frequency it can be simply modeled by a current sink and an equivalent load resistance using the Norton theorem. The current comes from the input FET, which acts as a transconductor transforming voltage signals upon its gate into current signals through its drain. Let us call " $\mathrm{g}_{\mathrm{m}}$ " its transconductance. The drain current signal flows mostly through the common base path of the cascode structure and reaches thereby the load resistance $R_{L}$. The open loop gain $\mathrm{A}_{0}$ is given by

$$
\mathrm{A}_{0}=\alpha \quad \mathrm{g}_{\mathrm{m}} \quad \mathrm{R}_{\mathrm{L}}
$$

where $\alpha$ is a loss factor which takes into account that a fraction of the FET current does not enter T1's emitter but flows in part through $\mathrm{R}_{\mathrm{D}}$ and in part through the Early resistance of the FET. In this case $\mathrm{g}_{\mathrm{m}} \approx 38 \mathrm{~mA} / \mathrm{V}$ and $\alpha$ is found to be in the range from 0.85 and 0.95 for the used bias points.

The load resistance is given by

$$
\mathrm{R}_{\mathrm{L}}=\mathrm{R}_{\mathrm{B} 3}\left\|\mathrm{R}_{\mathrm{I} 1}\right\| \mathrm{R}_{\mathrm{C} 2}
$$

where symbol " $\|$ " stands for "parallel connection of". $R_{\mathrm{B} 3}$ is the dynamic resistance looking into the base of transistor $\mathrm{T}_{3}, \mathrm{R}_{\mathrm{I} 1}$ is the dynamic resistance of active load $\mathrm{I}_{1}, \mathrm{R}_{\mathrm{C} 2}$ is the dynamic resistance looking into the collector of transistor $T_{2}$.

We are to maximize $R_{L}$ and so also $A_{0}$. For this purpose let us consider three cases: (a) single-transistor cascode and resistive load in place of $\mathrm{I}_{2}$, (b) single-transistor cascode and active load $\mathrm{I}_{2}$, (c) two-transistor cascode and active load $\mathrm{I}_{2}$. As can be seen
TABLE I

RELEVANT RESISTANCE VALUES

\begin{tabular}{cccccc}
\hline & $\mathrm{R}_{\mathrm{B} 3}$ & $\mathrm{R}_{\mathrm{II}}$ & $\mathrm{R}_{\mathrm{C} 2}$ & $\mathrm{R}_{\mathrm{L}}$ & $\mathrm{A}_{0}$ \\
\hline (a) & $312 \mathrm{k} \Omega$ & $4.92 \mathrm{M} \Omega$ & $487 \mathrm{k} \Omega$ & $183 \mathrm{k} \Omega$ & $6.22 \times 10^{3}$ \\
\hline (b) & $3.4 \mathrm{M} \Omega$ & $4.92 \mathrm{M} \Omega$ & $487 \mathrm{k} \Omega$ & $388 \mathrm{k} \Omega$ & $1.32 \times 10^{4}$ \\
\hline (c) & $3.4 \mathrm{M} \Omega$ & $4.92 \mathrm{M} \Omega$ & $1.68 \mathrm{M} \Omega$ & $917 \mathrm{k} \Omega$ & $3.03 \times 10^{4}$ \\
\hline
\end{tabular}

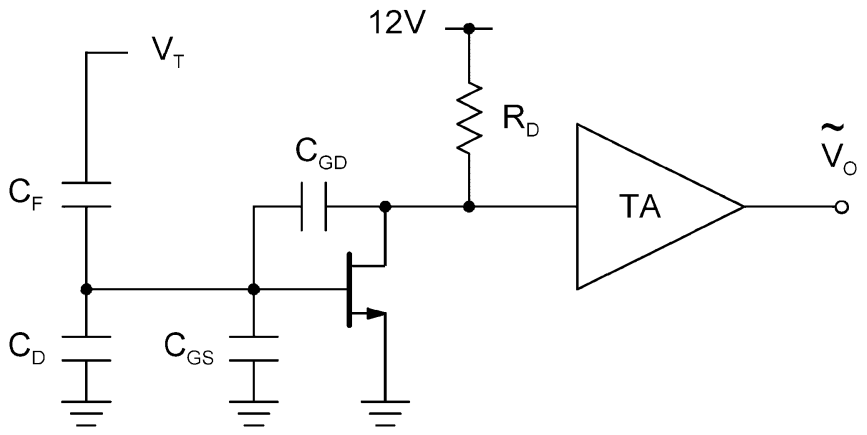

Fig. 5. Open-loop equivalent circuit used for the calculation of the loop gain The Transimpedance Amplifier structure is shown in Figs. 2(a) and Fig. 2(b). The capacitance values are shown in Table II.

in Table I, going from (a) to (b) yields an increased value of $R_{B 3}$. Going from (b) to (c) yields an increased value of $R_{C 2}$. So $A_{0}$ is improved step by step. In Table I the values of $R_{B 3}, R_{I 1}$, $\mathrm{R}_{\mathrm{C} 2}$ and $\mathrm{A}_{0}$ as obtained from a computer simulation are shown. The open-loop gain $A_{0}$ is found to be in good agreement with (1). The open-loop gain vs. frequency plot is shown in Fig. 4 for cases (a), (b) and (c). The dominant pole is due to capacitance $\mathrm{C}_{\mathrm{C}}$ and can be adjusted to ensure circuit stability. 


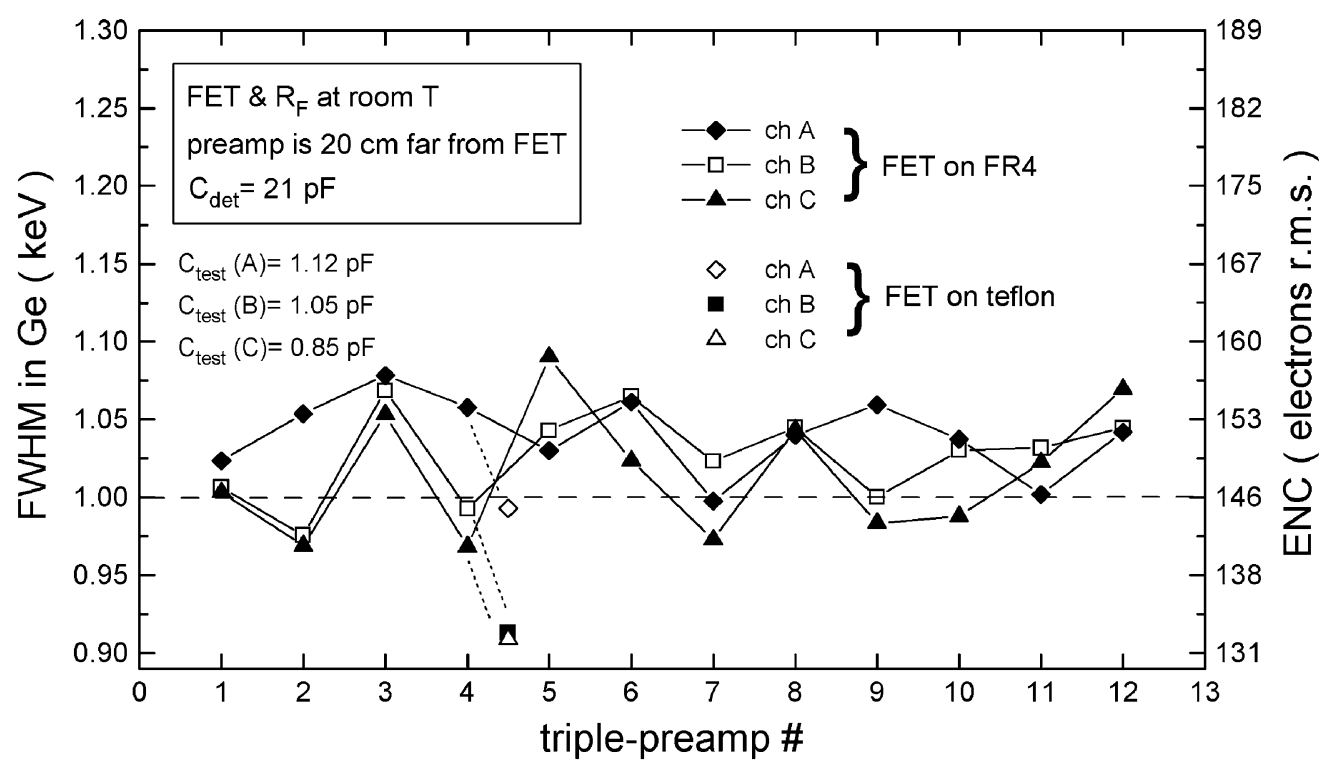

Fig. 6. Noise of preamplifier. A quasi-Gaussian shaper with $6 \mu$ s shaping time was used to filter the signal. The noise decreases when mounting the FET on a ptfe board.

TABLE II

RELEVANT CAPACITANCE VALUES

\begin{tabular}{cccc}
\hline $\mathrm{C}_{\mathrm{F}}$ & $\mathrm{C}_{\mathrm{D}}$ & $\mathrm{C}_{\mathrm{GS}}$ & $\mathrm{C}_{\mathrm{GD}}$ \\
\hline $1 \mathrm{pF}$ & $21 \mathrm{pF}$ & $4.4 \mathrm{pF}$ & $3.4 \mathrm{pF}$ \\
\hline
\end{tabular}

TABLE III

RELEVANT PARAMETERS

\begin{tabular}{ccccc}
\hline & $\mathrm{K}$ & $\mathrm{C}_{\mathrm{GD}}(1+\mathrm{K})$ & $\mathrm{G}_{0}$ & $\mathrm{~A}_{0} / \mathrm{G}_{0}$ \\
\hline (a) & 9 & $30.7 \mathrm{pF}$ & $1.1 \times 10^{2}$ & 57 \\
\hline (b) & 15 & $51.1 \mathrm{pF}$ & $1.7 \times 10^{2}$ & 78 \\
\hline (c) & 2.5 & $8.5 \mathrm{pF}$ & $0.84 \times 10^{3}$ & 36 \\
\hline
\end{tabular}

\section{B. Loop Gain and Miller Effect on $\mathrm{C}_{\mathrm{GD}}$}

In Fig. 5 the equivalent circuit used to calculate the loop gain $\mathrm{G}_{0}$ is shown. Taking into account the Miller effect acting on $\mathrm{C}_{\mathrm{GD}}, \mathrm{G}_{0}$ is easily obtained, or

$$
\begin{aligned}
G_{0} & =\frac{\tilde{V}_{o}}{V_{T}} \\
& =-\frac{C_{F}}{C_{F}+C_{D}+C_{\mathrm{GS}}+C_{\mathrm{GD}}(1+K)} A_{0}
\end{aligned}
$$

where $\mathrm{C}_{\mathrm{F}}$ and $\mathrm{C}_{\mathrm{D}}$ are the feedback and detector capacitances, $\mathrm{C}_{\mathrm{GS}}$ and $\mathrm{C}_{\mathrm{GD}}$ are the gate-to-source and gate-to-drain capacitances of the input FET, and $\mathrm{K}$ is the gate-to-drain voltage gain responsible for the Miller effect on $\mathrm{C}_{\mathrm{GD}}$. It can be seen that the loss term brought about by the capacitance divider decreases as $\mathrm{K}$ is increased. In Tables II and III the relevant parameters as obtained from a computer simulation are shown.

Note that going from (a) to (b) the gate-to-drain voltage gain $\mathrm{K}$ nearly doubles. Here is why: the increased value of the load resistance yields an increased value of the resistance looking

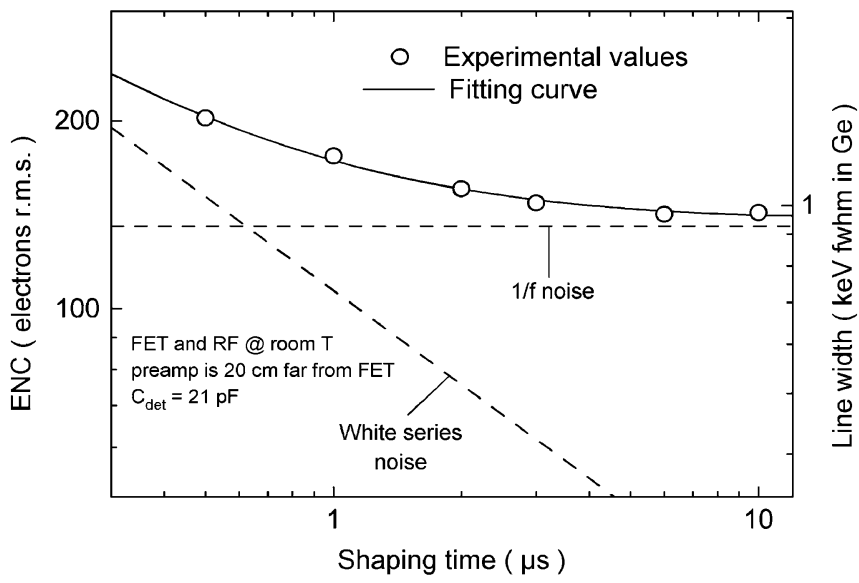

Fig. 7. Electronic noise versus shaping time.

into $T_{1}$ 's emitter, whose value ranges from $1 / g_{m}$ to $\beta / g_{m}$ when a high-value resistance is connected from its collector to ground [6]. The increased value of the resistance looking into $\mathrm{T}_{1}$ 's emitter yields in turn an increased value of $\mathrm{K}$ and a stronger Miller effect on $\mathrm{C}_{\mathrm{GD}}$. In order to reduce $\mathrm{K}$ the double-transistor cascode solution is introduced, which strongly reduces the value of the resistance connected from $\mathrm{T}_{1}$ 's collector to ground. The beneficial effect of such a circuit solution can be seen in Table II going from (b) to (c), where $\mathrm{K}$ drops substantially and $\mathrm{G}_{0}$ subsequently grows by almost one order of magnitude. The loop gain vs. frequency plot is shown in Fig. 4 for cases (a), (b) and (c).

\section{EXPERIMENTAL RESULTS}

We fully characterized the preamplifier at the test bench, using a capacitor to simulate the impedance of the detector electrode and a fast pulser to simulate the events. In Fig. 6 the noise of the preamplifier is shown for 36 different preamplifier channels, as measured operating the FET at room temperature and using a $21 \mathrm{pF}$ detector capacitance. The noise as a function 


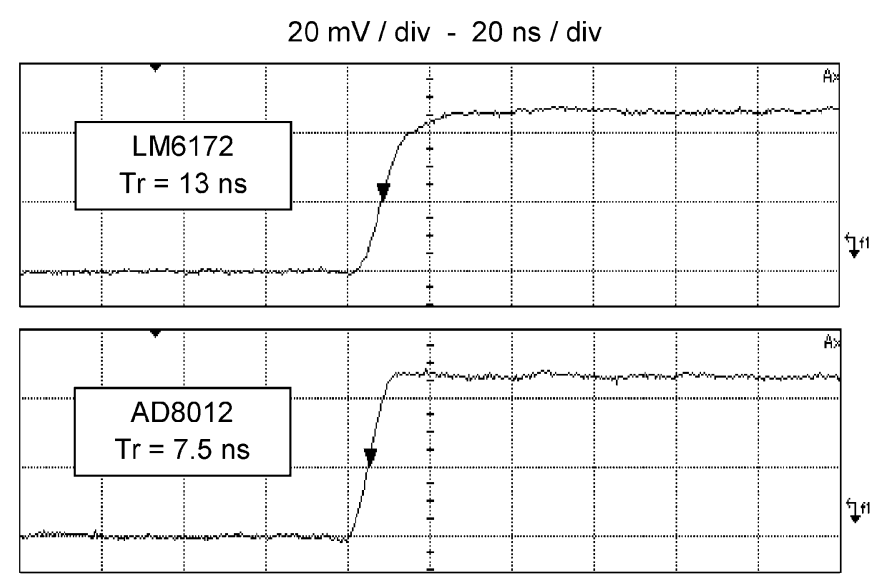

Fig. 8. Rise time of preamplifier. Results obtained at room temperature with $\mathrm{C}_{\text {det }}=21 \mathrm{pF}$.

TABLE IV

PREAMPLIFIER SPECIFICATIONS

\begin{tabular}{|c|c|}
\hline $\begin{array}{l}\text { Conversion gain }- \text { Ge det. } \\
\text { (terminated) }\end{array}$ & $100 \mathrm{mV} / \mathrm{MeV}+/-10 \mathrm{mV} / \mathrm{MeV}$ \\
\hline Rise time & $\begin{array}{c}7.5 \mathrm{~ns}(21 \mathrm{pF}) \\
\text { differential signal, } 5 \mathrm{~m} \text { terminated cable, } \\
\text { warm FET (mod. BF862) }\end{array}$ \\
\hline Slope & $\begin{array}{c}<0.4 \mathrm{~ns} / \mathrm{pF} \\
\text { (0 to } 79 \mathrm{pF} \text { range) }\end{array}$ \\
\hline Fall time & $50 \mu s+/-5 \%$ \\
\hline Noise & $\begin{array}{l}0.80 \text { keV fwhm (0 pF) @ } 3 \mu \text { s shap time* } \\
0.77 \text { keV fwhm (0 pF) @ } 6 \text { ss shap time* } \\
\text { "differential signal, warm FET (mod. BF862) }\end{array}$ \\
\hline Noise slope & $\begin{array}{c}7.7 \mathrm{eV} / \mathrm{pF} @ 3 \mu \mathrm{s} \text { shap time } \\
5.0 \mathrm{eV} / \mathrm{pF} @ 6 \mu \text { s shap time } \\
\text { (in } 0 \text { to } 68 \mathrm{pF} \text { range) }\end{array}$ \\
\hline $\begin{array}{l}\text { Power required } \\
\text { (including FET) }\end{array}$ & $\begin{array}{c}+12 \mathrm{~V} ; 8.5 \mathrm{~mA}\left(\mathrm{I}_{\mathrm{D}}=7.8 \mathrm{~mA}, \mathrm{~V}_{\mathrm{D}}=2.2 \mathrm{~V}\right) \\
-12 \mathrm{~V} ; 3.7 \mathrm{~mA} \\
+6 \mathrm{~V} ; 16 \mathrm{~mA} \\
-6 \mathrm{~V} ; 6.7 \mathrm{~mA} \\
\\
283 \mathrm{~mW}\end{array}$ \\
\hline Integral nonlinearity & $\begin{array}{l}0.04 \% \text { for a full scale range of }+/-3.5 \mathrm{~V} \\
\text { (unterminated) }\end{array}$ \\
\hline $\begin{array}{l}\text { Mechanical dimension } \\
\text { (for a triple preamplifier) }\end{array}$ & $\begin{array}{l}\quad 57 \times 56 \times 5 \mathrm{~mm} \\
\text { (including connectors) }\end{array}$ \\
\hline
\end{tabular}

of the shaping time is shown in Fig. 7. It reaches a minimum of about 140 electrons r.m.s. at a shaping time of $6 \mu \mathrm{s}$.

In the AGATA (Advanced GAmma ray Tracking Array) [1] setup the preamplifiers will be connected to sampling modules through long $50 \Omega$-terminated cables. In order to $(a)$ maintain a wide bandwidth while driving such a low impedance load, and $(b)$ guarantee an outstanding gain stability, use of a highquality output stage as operated in a closed-loop configuration is mandatory. The adopted output stage consists of a dual operational amplifier configured as a differential buffer, which helps reducing the common-mode disturbances picked up along the cable. The results are shown in Fig. 8 observing the signal after a $5 \mathrm{~m} 50 \Omega$-terminated twisted-pair cable. Two different dual operational amplifiers have been tested, both quoted for low

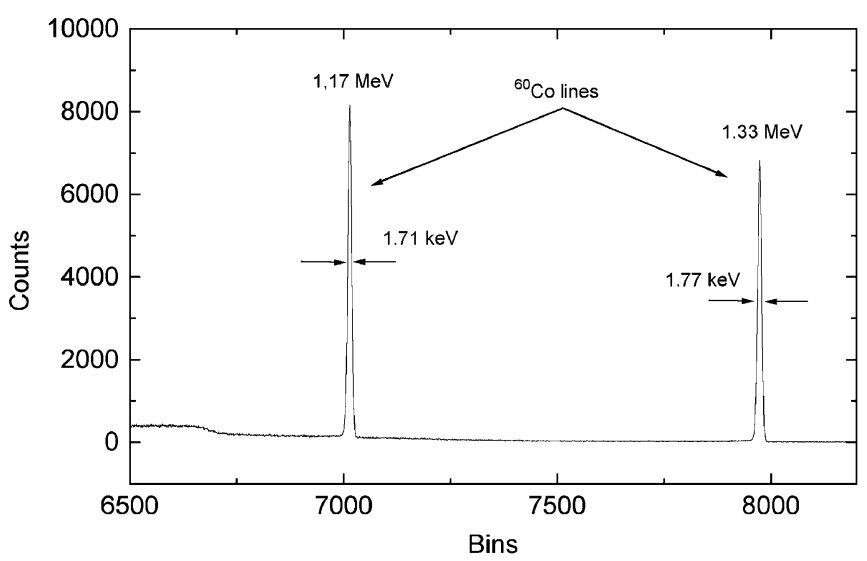

Fig. 9. Zoom of the $1.17 / 1.33 \mathrm{MeV}$ lines of ${ }^{60}$ Co spectrum.

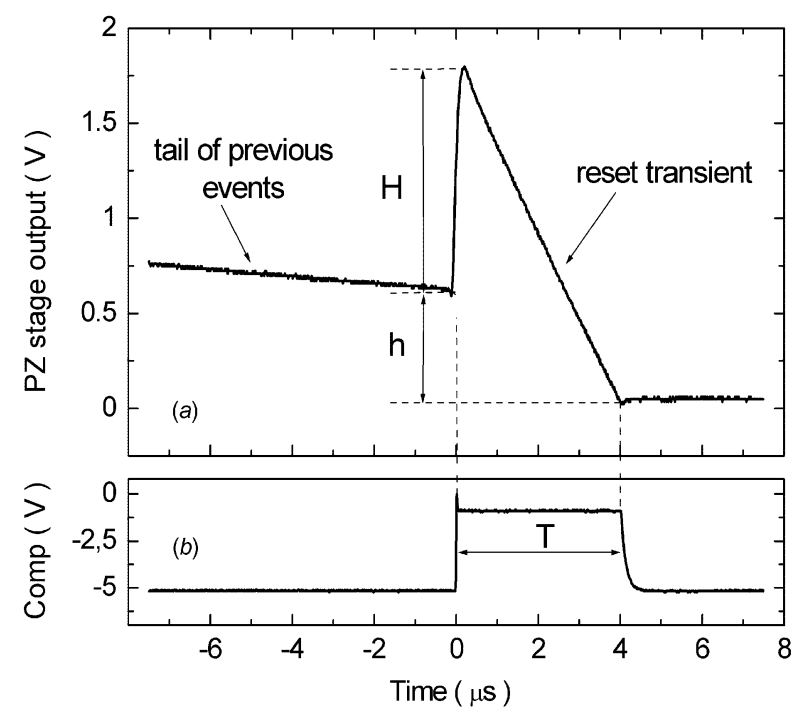

Fig. 10. (a) Large signal (H) upon a tail (h) at the PZ stage output. (b) Schmitttrigger comparator signal.

noise, low power and wide bandwidth. A rise time of $13 \mathrm{~ns}$ was obtained with the LM6172 and of as little as $7.5 \mathrm{~ns}$ with the AD8012. It is worth observing that the full bandwidth of the charge sensing stage can be exploited only if the output buffer maintains its performance when driving a low-impedance load.

If the detector capacitance is decreased from $21 \mathrm{pF}$ to $5-10 \mathrm{pF}$ as is typical for large multichannel systems, the loop gain (3) grows and the phase margin decreases. A ringing appears in the preamplifier response. However it can be easily removed stabilizing the feedback loop by increasing capacitance $\mathrm{C}_{\mathrm{C}}$ of Figs. 2(a) and (b), and so inherently reducing the gain-bandwidth product of the loop gain. In Table IV the specifications of the preamplifier are summarized.

Finally we connected the preamplifier to a cathode segment of an HPGe (High Purity Germanium) detector for the AGATA demonstrator [1] and collected the ${ }^{60}$ Co spectrum shown in Fig. 9. An excellent resolution was achieved of $1.71 / 1.77 \mathrm{keV}$ fwhm on the $1.17 / 1.33 \mathrm{MeV}$ lines. A resolution of $770 \mathrm{eV}$ fwhm was obtained on the $59 \mathrm{keV}^{241} \mathrm{Am}$ line, which is a good result for $\gamma$-ray spectroscopy systems. Of course better resolutions could be obtained at $59 \mathrm{keV}$ with 


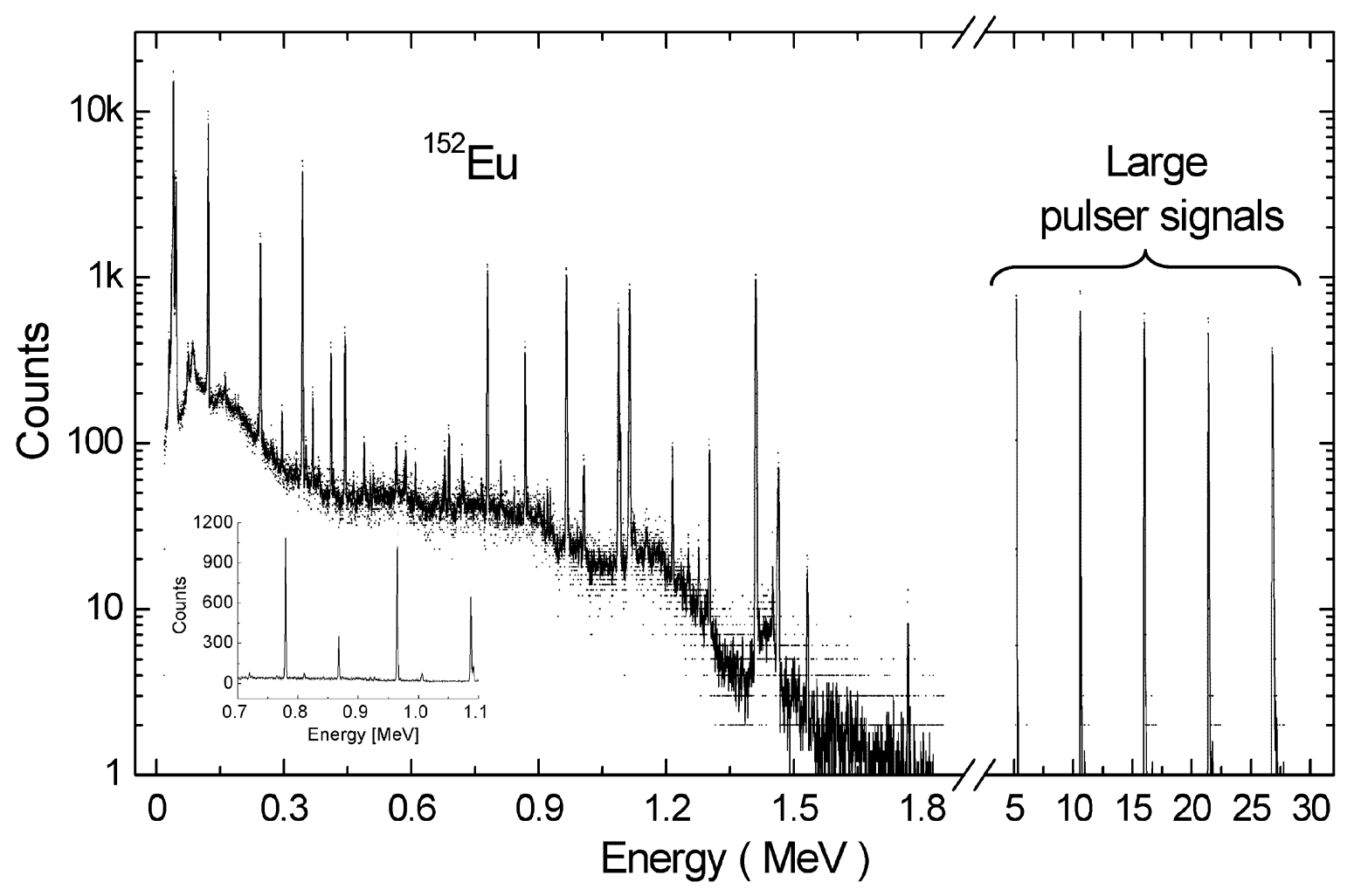

Fig. 11. Spectrum of ${ }^{152}$ Eu with a distribution of high-energy pulser lines. Note the scale break along the $x$ axis. The average event rate is $6 \mathrm{kHz}$.

preamplifiers designed mostly for X-ray spectroscopy, at the expense of a much narrower available energy range.

In the AGATA setup the power dissipation of the input FET, operated at cryogenic temperature, is as low as $17.1 \mathrm{~mW}$ (drain voltage $=2.2 \mathrm{~V}$, drain current $=7.8 \mathrm{~mA}$ ) which yields an overall power dissipation of $630 \mathrm{~mW}$ for each $37-\mathrm{elec}$ trode detector crystal. No micro-boiling of the cryogen has been observed. The inter-channel cross-talk has been reduced to less than $0.1 \%$ by carefully shielding the region where the detector electrode and the input FET are electrically connected.

\section{Reconstruction of LARge Signals From Reset Time}

The typical energy range of preamplifiers for $\gamma$-ray spectroscopy is from a few $\mathrm{keV}$ to a few $\mathrm{MeV}$. However in the next generation of nuclear physics experiments with exotic beams, a background of highly energetic charged particles (up to $100 \mathrm{MeV}$ ) is foreseen. Moreover, at high count rates, bursts of piled-up signals can easily exceed the typical energy range. These events cause ADC saturation and introduce a significant system dead time, which could even lead to a paralysis of the acquisition system.

The fast-reset device (see Fig. 3) has been implemented to address this issue. It yields a full reset of the preamplifier whenever it receives a large signal, i.e., a signal which overcomes a preset threshold, corresponding to the ADC saturation point. The reset time ranges from 0.5 to $150 \mu \mathrm{s}$, for signals in the 5 to $150 \mathrm{MeV}$ range. For signals below the threshold a normal resistive continuous reset is performed. As compared to pure continuous or pulsed reset solutions [7]-[9], such operation mode provides an improved performance: it maximizes the live time in the typical energy range and minimizes the dead time caused by amplitude saturation. Moreover it allows to measure the amplitude of large individual signals from the reset time, when the ADC overflow condition would give no possibility to make a direct measurement of the pulse height. As a result, the dynamic range is substantially increased.

In Fig. 10(a) we show the typical reset transient seen at the P/Z amplifier output (see Fig. 3), when a large individual signal (H) occurs upon a tail of a regular signal (h). Fig. 10(b) shows the corresponding waveform seen at the Schmitt-trigger comparator output, which delivers a rectangular pulse of width $\mathrm{T}$, corresponding to the fast-reset time. The regular signal which provides the tail $\mathrm{h}$ has the typical exponential decay time of about $50 \mu \mathrm{s}$. The large signal $\mathrm{H}$ is instead over threshold and puts the preamplifier into the fast reset mode. By accurately calculating the expression of the reset transient at the $\mathrm{P} / \mathrm{Z}$ stage output, we found an exponential relation between the reset time $\mathrm{T}$ and the sum of the energy $\mathrm{E}_{\mathrm{S}}$ of the large event and the energy $\mathrm{E}_{\mathrm{C}}$ of the tail, or

$$
E_{S}+E_{C}=A\left(e^{T / \tau}-1\right)
$$

where A and $\tau$ are coefficients depending on circuit parameters [5]. Because the reset time $\mathrm{T}$ is substantially shorter than the time constant $\tau$, the relation can be linearized with little loss of accuracy by expanding the exponential term. We can then express the large signal energy $\mathrm{E}_{\mathrm{S}}$ as a function of the reset time $\mathrm{T}$ and the energy of the tail $\mathrm{E}_{\mathrm{C}}$, obtaining

$$
\mathrm{E}_{\mathrm{S}}=\mathrm{b}_{1} \mathrm{~T}+\mathrm{b}_{2} \mathrm{~T}^{2}-\mathrm{E}_{\mathrm{C}}
$$




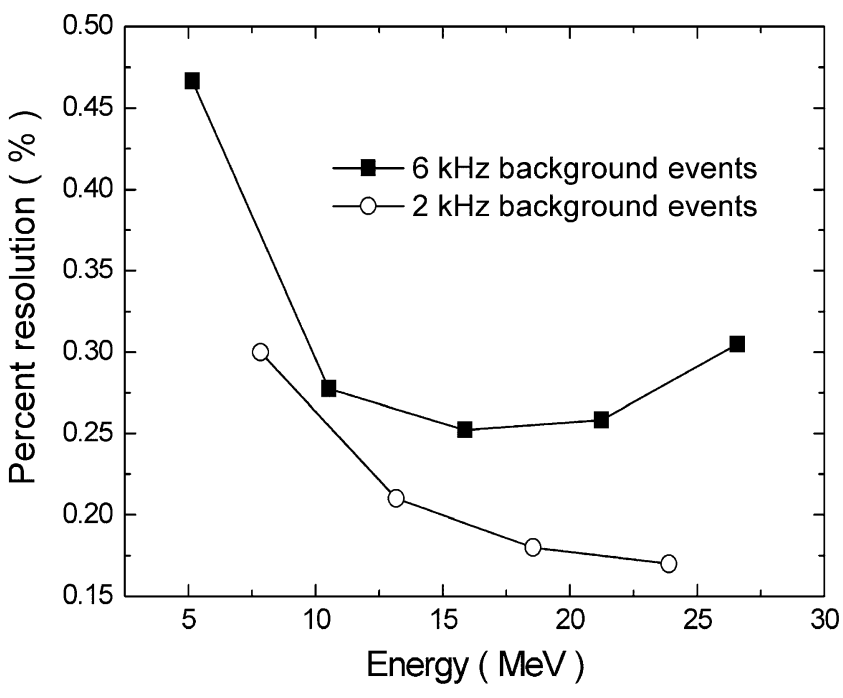

Fig. 12. Energy resolution obtained from the reset time at high energy for two different values of ${ }^{152}$ Eu "background" event rate.

where $b_{1}$ and $b_{2}$ are the the first- and second-order coefficients of the Taylor expansion. The reset time $\mathrm{T}$ can be obtained through a time over threshold measurement performed on the waveform acquired at the comparator output [see Fig. 10(b)], while the tail energy $E_{C}$ can be obtained from the difference of the pre- and post-large-event voltage levels evaluated on the output signal of the preamplifier. So (5) can be written as

$$
\mathrm{E}_{\mathrm{S}}=\mathrm{b}_{1} \mathrm{~T}+\mathrm{b}_{2} \mathrm{~T}^{2}-\left(\mathrm{V}_{1}-\mathrm{V}_{2}\right) / \mathrm{G}+\mathrm{E}_{0},
$$

where $(\mathrm{V} 1-\mathrm{V} 2)$ is the voltage amplitude of the tail, $\mathrm{G}$ is the energy-to-voltage gain of the preamplifier and $E_{0}$ is an offset term. We can conclude that (6) permits to indirectly derive the large signal energy $E_{S}$. In this relation $T, V_{1}$ and $V_{2}$ must be evaluated every time an over-threshold signal occurs, while $b_{1}, b_{2}, G$ and $E_{0}$ are parameters obtained through preliminary calibrations.

We tested this operation mode using an ORTEC coaxial HPGe detector [10], a calibration radioactive source and a pulser to simulate the arrival of energetic events. Fig. 11 shows a spectrum of ${ }^{152}$ Eu obtained while also injecting large pulser signals in the $20 \mathrm{MeV}$ range through a calibrated test capacitance. The ${ }^{152}$ Eu lines have been histogrammed using a quasi Gaussian shaper amplifier and a Pulse Height Analyzer, obtaining an energy resolution of $1.25 \mathrm{keV}$ fwhm on the $122 \mathrm{keV}$ line and $2 \mathrm{keV}$ fwhm on the $1.4 \mathrm{MeV}$ line. The large pulser signals, instead, have been histogrammed using the reset times and a proper calibration algorithm, according to (6). As can be seen, we could histogram very large signals of up to $27 \mathrm{MeV}$, enhancing the available preamplifier dynamic range by more than one order of magnitude as compared to the typical case. In Fig. 12 the energy resolutions obtained at high energy are shown for two different values of ${ }^{152}$ Eu "background" events.
Note that such a large dynamic range was obtained with no sacrifice in terms of energy resolution in the typical energy range for gamma spectroscopy. Namely we achieved a good energy resolution of about two per thousand in the $1 \mathrm{MeV}$ energy range and still a good energy resolution of about three per thousand in the $20 \mathrm{MeV}$ energy range. Such a large energy range, from $\sim 5 \mathrm{keV}$ up to $\sim 20$ or $30 \mathrm{MeV}$, can be of great interest for those experiments in which giant-dipole resonances up to $20 \mathrm{MeV}$ are to be measured.

\section{CONCLUSION}

A low-noise fast charge preamplifier purposely conceived for position-sensitive germanium detectors has been designed, constructed, and characterized. It comprises a fast-reset circuitry to reduce the dead time in the presence of a background of energetic events or large random walks. We could estimate the amplitude of the large events from the measurement of the reset time. The circuit structure of the preamplifier has been presented and discussed. We also carried out and presented an experimental characterization of the circuit as connected to a state-ofthe-art HPGe detector.

\section{ACKNOWLEDGMENT}

The authors would like to thank D. Bazzacco, R. Isocrate, C. Boiano, R. Bassini, and J. Eberth for their suggestions and stimulating discussions.

\section{REFERENCES}

[1] D. Bazzacco, B. Cederwall, J. Cresswell, G. Duchene, J. Eberth, and W. Gast, AGATA Tech. Proposal [Online]. Available: http://agata.pd. infn.it/documents/Agata-proposal.pdf

[2] I. Y. Lee, "Gamma-ray tracking detectors," Nucl. Instrum. Methods Phys. Res. A, vol. A422, pp. 195-200, 1999.

[3] Th. Kröll and D. Bazzacco, "Simulation and analysis of pulse shapes from highly segmented HPGe detectors for the gamma-ray tracking array MARS," Nucl. Instrum. Methods Phys. Res. A, vol. A463, pp. 227-249, 2001

[4] P. F. Bortignon, A. Bracco, and A. R. Broglia, Giant Resonances-Nuclear Structure at Finite Temperature. Australia: Harwood Academic Publishers, 1998.

[5] A. Pullia and F. Zocca, "A low-noise preamplifier for $\gamma$-ray sensors with add-on device for large-signal management," Nucl. Instrum. Methods Phys. Res. A, vol. A545, no. 3, pp. 784-92, 2005.

[6] J. Millman and A. Grabel, Microelectronics, 2nd ed. New York: McGraw-Hill, 1987, ch. 10.

[7] D. A. Landis, N. W. Madden, and F. S. Goulding, "Bipolar pulsed reset for AC coupled charge-sensitive preamplifiers," in Proc. IEEE Nuclear Science Symp. Conf. Rec., Albuquerque, NM, Nov. 9-15, 1997, vol. 1, pp. 887-891.

[8] T. Lakatos, G. Hegyesi, and G. Kalinka, "A simple pulsed drain feedback preamplifier for high resolution high rate nuclear spectroscopy," Nucl. Instrum. Methods Phys. Res. A, vol. 378, no. 3, pp. 583-588, 1996.

[9] D. A. Landis, C. P. Cork, N. W. Madden, and F. S. Goulding, "Transistor reset preamplifier for high resolution spectroscopy," IEEE Trans. Nucl. Sci., vol. NS-29, no. 1, pp. 619-624, 1982.

[10] Th. Kröll, D. Bazzacco, R. Venturelli, B. Quintana Arnes, and C. A Ur, "Gamma-ray tracking with the MARS detector," Eur. J. Phys., vol. 20, no. 1, pp. 205-206, 2003. 